\title{
THE EFFECT OF THE POLYSACCHARIDE FRACTION FROM Hericium erinaceus ON POTATO IN VITRO AND EX VITRO
}

\author{
Bakulina Anna, Nazarova Yanina, Shirokikh Irina, Shirokikh Al. \\ Federal Agricultural Research Center of the North-East named N.V. Rudnitsky, Russia \\ e-mail: drugaeann1@rambler.ru
}

Culture of plant cells and tissues is the basis of traditional (clonal micropropagation, cell selection, etc.) and modern (genetic engineering, genome editing) methods of agrobiotechnology. When cultivated in vitro, plant cells are under stress, and the actual problem of plant biotechnology is still the search for substances that have a positive effect on plant cells and tissues, which contribute to reducing stress, as well as increase the regeneration of plants from explants. Among the variety of substances that have physiological activity against cells of living organisms, polysaccharides of basidial fungi are of interest, which are now widely used in medicine, but their effect on plant tissues has not been previously studied. The aim of this work was to study the effect of the polysaccharide fraction (PF) of fruiting bodies of basidiomycetous mushroom Hericium erinaceus on the morphological, biochemical characteristics and productivity of potato during its cultivation in vitro and ex vitro.

The object of the study was H. erinaceus BP19 PF №4, isolated by extraction with water at $70^{\circ} \mathrm{C}$ (Nazarova et al., 2018) from dry fruit bodies obtained by cultivation of the fungus in the laboratory of biotechnology of plants and microorganisms FARC North-East. Identification of the natural isolate BP19 was carried out by sequencing of ITS1-5.8S-ITS2 region (GenBank Accession number MK809367). We used potato variety of Pransa. In vitro meristem plants were grown on Murashige-Skoog medium without hormones. In passage to fresh medium nodal cuttings dipped in a solution of PF $(0.5 \mathrm{mg} / \mathrm{ml})$ in control PF not used. Experimental and control plants were cultivated for 7 weeks then transferred to ex vitro.

Treatment of potatoes with PF from $H$. erinaceus BP19 virtually no influence on morphometric parameters microplants, causing a slight (7.0 and 9.0\%) compared to the control elongation of the stem 20 and 30 days of cultivation. At the same time, the study of biochemical parameters of potatoes in vitro exposed to $\mathrm{PF}$ revealed a decrease in the level of lipid peroxidation in treated PF plants relative to the control (MDA content of $14.7 \pm 1.33$ and $18.8 \pm 1.58 \mathrm{nmol} \mathrm{g}^{-1}$ dry wt., respectively). The evaluation of this parameter in ex vitro plants show that after 4 weeks of growing in the soil, peroxide homeostasis of plants exposed to PF treatment did not differ from that in the control, but significantly decreased after 5 and 6 weeks of growing: the MDA content in leaf tissues in experimental plants was $8.1 \pm 0.64$ and $9.3 \pm 0.30 \mathrm{nmol} \mathrm{g}^{-1}$ dry wt., and in control plants $-9.7 \pm 0.80$ and $10.2 \pm 0.55 \mathrm{nmol} \mathrm{g}^{-1}$ dry wt. In addition, differences in potato productivity were revealed. Plants treated with PF were characterized by an increase in the number of minitubers per plant $(3,6 \pm 0,20)$ in comparison with the control (2.0 \pm 0.67$)$, as well as an increase in their size: the fraction $\geq 40 \mathrm{~mm}$ increased to $28 \%$, while in the control was only $22 \%$. It was revealed a significant increase in the average yield per plant as a result of PF treatment (48.6 $\pm 9.06 \mathrm{~g})$ in comparison with the control $(25.0 \pm 1.37 \mathrm{~g})$.

Thus, on the example of potatoes it was found that $\mathrm{PF}$ from $H$. erinaceus $\mathrm{BP} 19$ has a positive effect on plant tissue. According to the biochemical assessment, the treated PF plants were less stressed than the control plants in vitro and ex vitro, and the potato productivity under the influence of PF increased 1.9 times. The data obtained indicate the need for further study of the effect of PF from $H$. erinaceus on plants, it is planned to assess the activity of PF on the callus culture of monocotyledons, for which the search for substances - inducers of morphogenesis is particularly relevant. 